\title{
Current-carrying Capacity of Carbon Nanofiber Interconnects
}

\author{
Hirohiko Kitsuki, Tsutomu Saito ${ }^{*}$, Toshishige Yamada, Drazen Fabris, John R. Jameson, \\ Patrick Wilhite, Makoto Suzuki, and Cary Y. Yang \\ Center for Nanostructures, Santa Clara University \\ Santa Clara, California 95053-0569, USA \\ *Corresponding author, phone: +1-408-554-6817, fax: +1-408-554-5474, e-mail: tsaito1@scu.edu
}

\begin{abstract}
Current-carrying capacity of carbon nanofibers (CNF) is investigated for potential interconnect applications. The measured maximum current density in the suspended CNF in air is inversely proportional to nanofiber length and independent of diameter. This relationship can be described with a heat transport model that takes into account Joule heating and heat diffusion along the CNF, assuming that breakdown occurs when and where the temperature reaches a threshold or critical value. Supporting the CNFs with $\mathrm{SiO}_{2}$ improves their current capacity, which implies effective heat dissipation to the oxide.
\end{abstract}

\section{Introduction}

Because of their high electrical and thermal conductivities as well as current capacity compared to conventional metals, carbon-based nanostructures such as carbon nanotubes $(\mathrm{CNTs})^{1-5}$ and carbon nanofibers $(\mathrm{CNFs})^{6.7}$ are being investigated for high-performance device and interconnect applications. The growth of CNFs consistently yields high conductivity and high directionality, which are attractive for realistic interconnect fabrication processes ${ }^{6}$. In our previous study ${ }^{7}, \mathrm{CNF}$ vias embedded in $\mathrm{SiO}_{2}$ demonstrated high degree of reliability while being subjected to a stressing current of $1 \times 10^{7} \mathrm{~A} / \mathrm{cm}^{2}$. Thus such a structure is expected to achieve the current density target set by the International Technology Roadmap for Semiconductors (ITRS) ${ }^{8}$ for the year 2015.

In CNT systems, breakdown phenomena have been observed under high electric fields, including nonlinear transport in single-walled $\mathrm{CNTs}^{9}$ and successive graphitic wall breakdown in multi-walled CNTs ${ }^{10}$. In our recent studies $^{7,11}$, proof of concept of the high-current reliability of CNFs for on-chip interconnects and the high-field transport properties of CNFs have been demonstrated. These results indicate that CNF breakdown mainly depends on resistive heating ${ }^{12}$, but details of the failure mode due to high current and the accompanying physical breakdown mechanisms have yet to be investigated. The significance of Joule heating in current-induced breakdown ${ }^{11}$ implies that thermal contact coupling between the CNF, electrodes, and surrounding materials (e.g., $\left.\mathrm{SiO}_{2}\right)$ affects the current capacity of the CNF. Meanwhile, current annealing has been reported to drastically reduce the overall resistance and was attributable to significant lowering of the contact resistances in CNT devices $^{13}$. Therefore, it is reasonable to assume that durability of CNF under high-current stress is largely dependent on the behavior of the nanofiber resistance during the Joule heating process. In this work, systematic characterization of CNFs under high-current stress is performed to examine and elucidate this behavior.

\section{Experiment}

The CNF samples are grown by plasma-enhanced chemical vapor deposition (PECVD) with a Ni catalyst layer on $\mathrm{Si}$ substrate. The detailed growth conditions have been described elsewhere ${ }^{14}$. A solution of CNFs is prepared by dispersion into a solvent of isopropyl alcohol. The solution is subjected to ultra-sonication and centrifuge in order to separate the CNFs into individual, well-dispersed structures. The solution is then drop-cast onto a substrate of prepatterned gold electrodes on an oxidized silicon wafer. Figure 1(a) shows a CNF sample suspended between gold electrodes, with a SEM image at $75^{\circ}$ tilted-angle view. This planar geometry is a model of horizontal on-chip interconnect configuration, where the CNF sidewall is in contact with the electrodes ${ }^{15}$. A DC current source connected to these electrodes is also shown in Fig. 1(a). Constant-current stress (equivalent to current annealing) is then carried out to monitor the electrical resistance prior to breakdown in air. In order to study the failure mode of the CNFs fabricated in a more realistic device structure on a $\mathrm{Si}$ substrate, $\mathrm{CNFs}$ supported by $\mathrm{SiO}_{2}$ are also examined, as shown in Fig. 1(b). Results for twenty devices, with CNFs ranging from 100 to $200 \mathrm{~nm}$ in diameter and 1.5 to $6 \mu \mathrm{m}$ in length, are presented in this paper.

The progression of constant-current stress cycles (at 180 sec. each) is illustrated in Figure 2(a). At the end of each cycle, $I-V$ characteristics are obtained around $V=0$. Figure 2(b) shows the resistance of a CNF device after each annealing cycle versus annealing current. Increasing the annealing current results in a gradual decrease in resistance before the nanofiber breaks down, at $700 \mu \mathrm{A}$ for this particular device. Since the measured resistance represents the sum of bulk and contact resistances and the nanofiber consistently breaks away from the contacts for suspended CNFs (see next section), this result suggests that current annealing likely reduces the contact resistances significantly, while the device approaches breakdown due to resistive Joule heating in the bulk of the CNF.

In our previous study ${ }^{11}$, systematic analysis using scanning transmission electron microscopy (STEM) and in situ SEM measurements has revealed the creation of void and 
defective graphitic layers in the CNF induced by current stress. This analysis suggested that the CNF resistance just before breakdown increases drastically due to severe degradation of the nanofiber internal structure, consistent with our present observation.
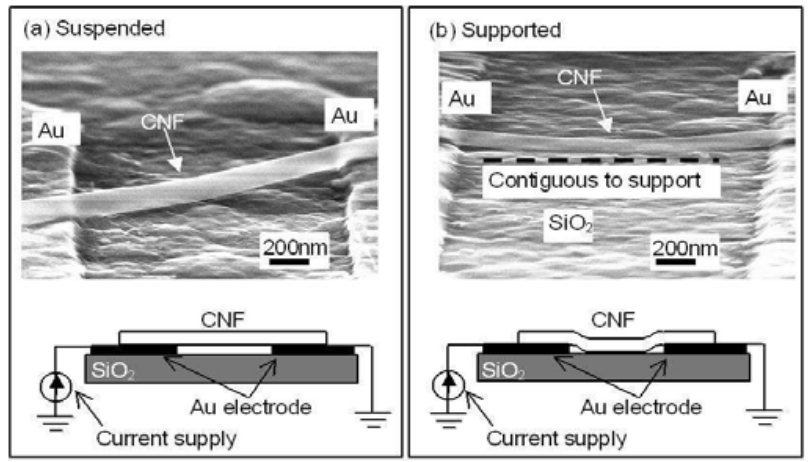

Fig. 1 Set-up for current-stressing experiments. (a) CNF suspended by gold electrodes. (b) $\mathrm{CNF}$ supported by $\mathrm{SiO}_{2}$ substrate. Upper figures show SEM image of a CNF sample at $75^{\circ}$ tilted-angle view, lower figures illustrate schematic of electrical measurement.

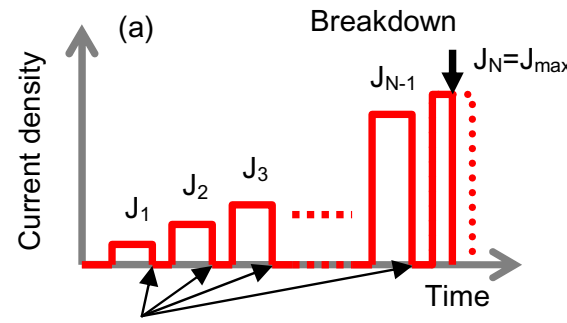

IV measurement around $\mathrm{V}=0$

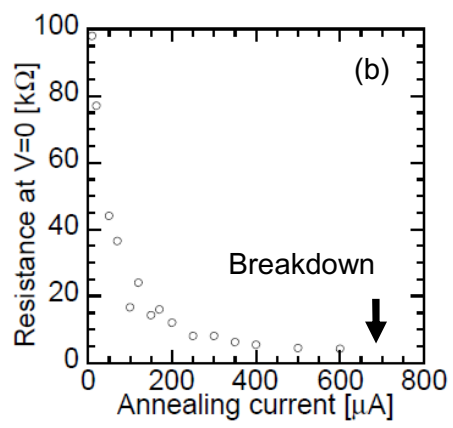

Fig.2 Resistance reduction of CNF device due to current annealing. (a) Schematic of successive current annealing cycles using stepwise increment of stressing current. (b) Resistance of the CNF device at $V=0$ obtained after each annealing cycle.

\section{Results and Discussion}

SEM image of a CNF suspended by gold electrodes before and after current-induced breakdown is shown in Figure 3(a). In all experiments for suspended CNFs, we have confirmed that breakdown always occurs near the middle of the nanofiber. This is consistent with diffusive heat transport observed in CNTs at high bias ${ }^{10}$, suggesting that resistive heating $^{12}$ is critical to the breakdown of CNFs. Figure 4 shows the relationship between the maximum current density $\left(J_{\max }\right)$ and the reciprocal of CNF length. Data obtained for the suspended CNF are given by solid circles. The decrease of $J_{\max }$ with increasing length is consistent with current-induced breakdown of single-walled carbon nanotubes (SWNT) ${ }^{16}$ and gold nanowires fabricated using conventional lithography ${ }^{17}$. This observed behavior is useful for predicting the current capacity of CNFs with different lengths when designing interconnects.
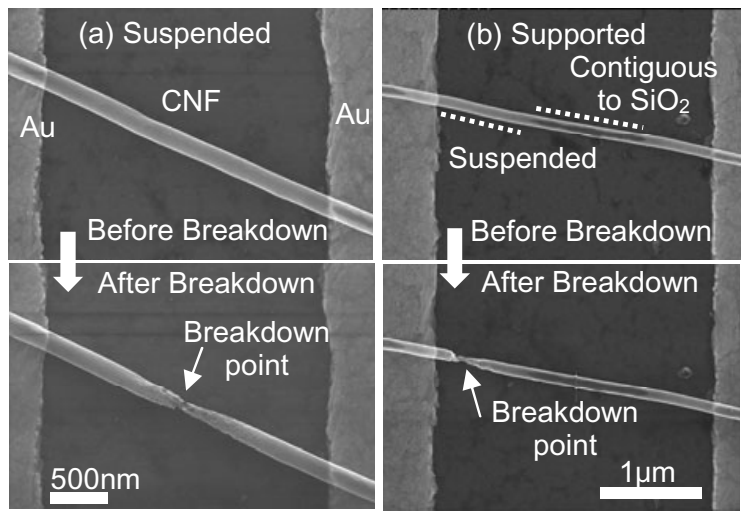

Fig. 3 SEM images of CNFs before and after current stressing at the top view. (a) CNF suspended by gold electrode. (b) CNF supported by $\mathrm{SiO}_{2}$ substrate.

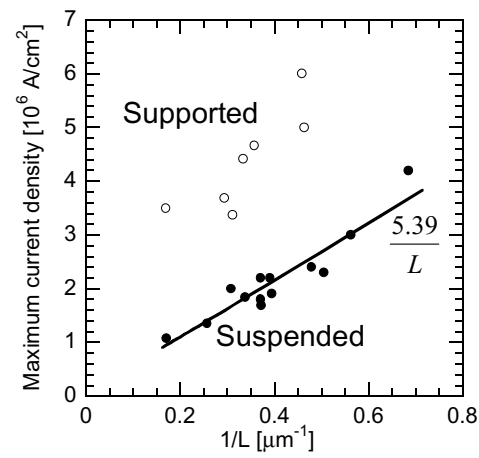

Fig. 4 Dependence of maximum current density on CNF length obtained using 20 devices. The solid and open circles show the results for suspended and supported CNFs, respectively. The straight line is a linear fit for suspended CNFs, as predicted by the heat transport model.

To understand the observed current capacity versus length behavior for the suspended CNF, a one-dimensional thermal transport model ${ }^{12,18}$ is used. We define $\Delta T(x)$ as the difference between the local temperature $T(x)$ and the temperature $T_{\infty}$ at infinity. $\Delta T(x)$ is determined through the balance among heat diffusion $\left(d^{2} \Delta T / d x^{2}\right)$, heat dissipation to the substrate $\left(a^{2} \Delta T\right)$, and heat generation due to Joule heating $(f)$, using

$$
\frac{d^{2} \Delta T}{d x^{2}}-a^{2} \Delta T=-f
$$

Here $f=I^{2} /\left(A^{2} \sigma \kappa\right)$, where $I$ is the current, $A$ the crosssectional area, $\sigma$ the electrical conductivity, and $\kappa$ the $\mathrm{CNF}$ thermal conductivity. Also $a^{2}=w \gamma A \kappa$, where $w$ is the effective contact line width and $\gamma$ is a coupling coefficient to account for the efficiency of heat transport through the CNF surroundings. We choose the origin $x=0$ at the midpoint of the fiber, with the two ends located at $x= \pm L / 2$. We impose the boundary conditions $\Delta T=0$ at $x= \pm L / 2$. The solution is then given by $\Delta T(x)=\left(f / a^{2}\right)[1-\cosh (a x) / \cosh (a L / 2)]$, with the maximum occurring at $x=0$. We assume that breakdown occurs when $T$ reaches a threshold or critical temperature $T_{\max }$, 
which is common for all CNFs with any diameters or lengths, obtained using the same growth process. Thus, $J_{\max }$ can be obtained as a function of $L$,

$$
J_{\max }=\left[\left(T_{\max }-T_{\infty}\right) \sigma \not w / A\right]^{1 / 2}[1-1 / \cosh (a L / 2)]^{-1 / 2}
$$

In the suspended case, heat dissipation is expected to be negligible, or $a L<<1$. In this limit,

$$
J_{\max } \approx 2 \sqrt{2\left(T_{\max }-T_{\infty}\right) \sigma \kappa} / L .
$$

In Figure 4, the measured $J_{\max }$ is plotted as a function of $1 / L$. It is seen that the $J_{\max }$ versus $1 / L$ behavior can be fitted to a straight line, as predicted by Eq. (3). This agreement confirms that for a suspended $\mathrm{CNF}$, heat dissipation to the surroundings (air) is small, and heat conduction along the length of the CNF causes the highest temperature to occur at the middle of CNF. It also verifies that $J_{\max }$ scales with $1 / L$, consistent with the peak current behavior of SWNTs ${ }^{16}$.

Combining the experimentally fitted line for $J_{\max }$ versus $1 / L$ in Figure 4 with Eq. (3), the critical temperature $T_{\max }$ predicted by the simple heat transfer model is estimated to be $1260 \mathrm{~K}$. This is comparable to the CNF synthesis temperature, generally in the $1000 \mathrm{~K}$ range ${ }^{19}$. In computing the critical temperature, a CNF thermal conductivity of $12 \mathrm{~W} / \mathrm{m} \mathrm{K}^{20}$ and the maximum electrical conductivity from the present data are used. Given the simplifications of this model, having no radiative heat transfer and in the limit of no coupling with the substrate, this estimate is only an indication of CNF durability and points to the need for systematic local temperature measurement.

For CNFs supported by $\mathrm{SiO}_{2}, \mathrm{SEM}$ images before and after current-induced breakdown are shown in Figure 3(b). The interface between CNF and the substrate is imaged using SEM contrast ${ }^{21}$. A dark-contrast region along the CNF indicates a section of the nanofiber contiguous to the substrate, while the bright section is not in contact or suspended. Breakdown occurs where CNF is suspended above the substrate. The maximum current density observed for the supported configuration is plotted with open circles in Figure 4. The supported CNFs show significantly improved current capacity in comparison to the suspended CNFs. However, the dependency of maximum current density on length is not as clear, due to variations in contact area between the different devices, which in turn result in variations in heat dissipation to the substrate. This finding indicates that heat dissipation via its immediate surroundings is a critical determining factor for high-current transport in CNF.

\section{Conclusion}

Current-induced breakdown was investigated for suspended CNFs. A simple relationship between the maximum current capacity and CNF length was deduced from heat transport modeling and verified by measurement results. This result suggests that the breakdown occurred when the local temperature of the nanofiber reached a dimension-independent critical temperature. The dependence of CNF current capacity on length is consistent with metallic nanowires, and the analysis presented here represents an important step toward the understanding of the reliability of CNF for potential interconnect applications.

\section{Acknowledgment}

The authors are grateful to Jun Li of Kansas State University and Alan M. Cassell of NASA Ames Research Center for their helpful advice. This work is supported by the U.S. Department of the Army.

\section{References}

[1] R. Martel, T. Schmidt, H.R. Shea, T. Hertel, P. Avouris, Appl. Phys. Lett. Vol. 78, 2447-2449 1998

[2] A. Javey, J. Guo, Q. Wang, M. Lundstrom, H. Dai, Nature, Vol. 424, 654-657 2003.

[3] J. Hone, I. Ellwood, M. Muno, A. Mizel, M.L. Cohen, A. Zettl, A.G. Rinzler, R.E. Smalley, Phys. Rev. Lett., Vol. 80, 1042 - 10451998.

[4] T. Yamada, Phys. Rev. B, Vol. 69, 1254082004.

[5] M. Nihei, M. Horibe, A. Kawabata, Y. Awano, Japan. J. Appl. Phys., Vol. 43, 1856-1859 2004.

[6] J. Li, Q. Ye, A.M. Cassell, H.T. Ng, R. Stevens, J. Han, M. Meyyappan, Appl. Phys. Lett., Vol. 82, 2491-2493 2003.

[7] Q. Ngo, A.M. Cassell, A.J. Austin, J. Li, S. Krishnan, M. Meyyappan, C.Y. Yang, IEEE Electron Device Lett., Vol. 27, 221-224 2006.

[8] International Technology Roadmap for Semiconductors (ITRS) 2006 Update. [Online]. Available: http:/public.itrs.net

[9] E. Pop, D.A. Mann, J. Cao, K.E. Goodson, H. Dai, J. Appl. Phys., Vol. 101, 0937102007.

[10] J.Y. Huang, S. Chen, S.H. Jo, Z. Wang, D.X. Han, G. Chen, M.S. Dresselhaus, Z.F. Ren, Phys. Rev. Lett., Vol. 94, 2368022005.

[11] M. Suzuki, Y. Ominami, Q. Ngo, C.Y. Yang, J. Li, A.M. Cassell, J. Appl. Phys., Vol. 101, 1143072007.

[12] M.A. Kuroda, A. Cangellaris, J.-P. Leburton, Phys. Rev. Lett., Vol. 95, 2668032005.

[13] J.-O Lee, C. Park, J.-J. Kim, J. Kim, J.W. Park, K.-H. Yoo, J. Phys. D., 33, 1953-1956 2000.

[14] B.A. Cruden, A.M. Cassell, Q. Ye, M. Meyyappan, J. Appl. Phys., Vol. 94, 4070-4078 2003.

[15] L. Zhang, D. Austin, V.I. Merkulov, A.V. Meleshko, K.L. Klein, M.A. Guillorn, D.H. Lowndes, M.L. Simpson, Appl. Phys. Lett. Vol. 84, 3972-39742004.

[16] E. Pop, D. Mann, J. Cao, Q. Wang, K. Goodson, H. Dai, Phys. Rev. Lett., Vol. 95, 1555052005.

[17] C. Durkan, M.A. Schneider, M.E. Welland, J. Appl. Phys., Vol. 86 , 1280-1286 1999

[18] H.S. Carslaw, J.C. Jaeger, Conduction of Heat in Solids Second Edition; Oxford University Press, Oxford, 1986.

[19] K.B.K. Teo, D.B. Hash, R.G. Lacerda, N.L. Rupesinghe, M.S. Bell, S.H. Dalal, D. Bose, T.R. Govindan, B.A. Cruden, M. Chhowalla, G.A.J. Amaratunga, M. Meyyappan, W.I. Milne, Nano Lett., Vol. 4, 921-926, 2004.

[20] C. Yu, S. Saha, J. Zhou, L. Shi, A.M. Cassell, B.A. Cruden, Q. Ngo, J. Li., J. Heat Transfer, Vol. 128, 234-239 2006

[21] M. Suzuki, Y. Ominami, Q. Ngo, C.Y. Yang, T. Yamada, J. Li, A.M. Cassell, J. Appl. Phys., 100, 1043052006. 